\title{
Social, cultural and community engagement and mental health: cross-disciplinary, co-produced research agenda
}

Daisy Fancourt, Kamaldeep Bhui, Helen Chatterjee, Paul Crawford, Geoffrey Crossick, Tia DeNora and Jane South

\section{Background}

There is increasing cross-disciplinary research on the relationship between individuals' social, cultural and community engagement (SCCE) and mental health. SCCE includes engagement in the arts, culture and heritage, libraries and literature, sports and nature activities, volunteering, and community groups. Research has demonstrated the effects of these activities both on the prevention and management of mental illness. However, it remains unclear whether current research is focusing on the research questions that are of most immediate urgency and relevance to policy and practice.

\section{Aims}

The current project was funded as part of the UK Research and Innovation cross-disciplinary mental health network programme to develop and co-produce a new cross-disciplinary research agenda on SCCE and mental health.

\section{Method}

Established processes and principles for developing health research agendas were followed, with a six-phase design including engagement with over 1000 key stakeholders, consultations, integration of findings and collective prioritisation of key questions.

\section{Results}

We identified four core themes: the mode of engagement, process of engagement, impact of engagement and infrastructure required to facilitate engagement. There were many points of agreement across all stakeholder groups on the priority questions within these themes, but also some specific questions of relevance to different sectors.

\section{Conclusions}

This agenda is particularly timely given the extreme pressure on mental health services predicted to follow the current COVID-19 pandemic. It is important to identify how resources from other sectors can be mobilised, and what research questions are going to be most important to fund to support SCCE for mental health.

\section{Keywords}

Psychosocial interventions; community mental health; research agenda; social; cultural.

\section{Copyright and usage}

(c) The Author(s), 2020. Published by Cambridge University Press on behalf of the Royal College of Psychiatrists. This is an Open Access article, distributed under the terms of the Creative Commons Attribution licence (http://creativecommons.org/ licenses/by/4.0/), which permits unrestricted re-use, distribution, and reproduction in any medium, provided the original work is properly cited.

\section{Community assets and mental health}

Over the past decade, there has been a growing focus on the resources (or 'assets') that exist within communities and how they can support health and well-being. ${ }^{1}$ Community assets in the broadest sense can include the skills and knowledge of individual community members, local groups and community and voluntary associations; the public-, private- and third-sector resources and facilities within communities; and physical, environmental and economic resources. ${ }^{1}$ Action to surface and mobilise such community resources for health and well-being as well as other purposes is sometimes referred to as an 'asset-based approach'.,

One of the areas of asset-based research that has received increasing attention in recent years is the role of community assets in leisure behaviours, including enabling and determining people's social, cultural and community engagement (SCCE). SCCE can include activities that require active participation (e.g. participating in arts, literature, sports, volunteering, nature activities or community groups) and those that involve a broader type of engagement with community assets (e.g. visiting heritage sites, museums, libraries and spending time outdoors in green spaces). More specific examples of SCCE are shown in Table 1.

There are estimated to be over 1 million assets within communities in the UK that support these types of SCCE: over 40000 choirs, 11000 amateur orchestras, 50000 amateur arts groups, 5000 amateur theatre societies, 3000 dance groups, 2500 museums, 400 historic places, 4000 libraries, 1300 theatres, 50000 book clubs, 27000 public parks, 1000 community gardens, 6500 leisure centres, 151000 sports clubs, 10000 village halls, 330000 allotments, 161000 voluntary associations and 160000 community groups. ${ }^{4}$ These assets are generally shaped and funded not by the health or social care sector, but by the creative, cultural, community and environmental sectors and by communities themselves. However, they have been shown to act as in-kind resources to health and social care through the impact they can have on mental health. From a prevention perspective, there is a rich research literature on SCCE and hedonic, eudemonic, social wellbeing and the prevention of mental illness. ${ }^{5-11}$ From a treatment perspective, specific programmes involving SCCE have also been developed for a wide range of mental illnesses, including psychotic disorders, addiction recovery, mood disorders, anxiety disorders and personality disorders. ${ }^{7,12-15}$ Further, we have an increasingly sophisticated understanding of why we find these effects, with a recent review identifying over 600 mechanisms of action. ${ }^{16}$ Some of the best-evidenced mechanisms include psychological processes (such as supporting emotion regulation, building resilience, developing identity and enhancing cognitive resources), biological processes (such as modulating brain activity, altering levels of hormones, increasing physical performance and mitigating biological weathering), social processes (such as increasing social connections, developing supportive relationships and enhancing social cohesion) and behavioural processes (such as increasing 

drawing/woodwork/painting/photography/ceramics/sculpture)

Culture and heritage

Going to museums, galleries or exhibitions; the theatre or concerts; the cinema; festivals, fairs or events; stately homes or buildings; historical sites; and landscapes of significance

Libraries and literature Going to libraries, visiting archives and being a member of book clubs or writing groups

Sports and nature

activities

Going to parks or gardens, engagement with allotments or gardening groups, joining nature walks or rambling groups, participation in exercise classes, membership of sports clubs, participating in community sporting activities and attending amateur or professional sporting events

Volunteering

Community groups 
commissioning or strategy. We then recruited 93 individuals within these different groups to join our research network as founding members and asked them to identify and recruit further members by using a snowballing approach. This led to a total recruitment of 1000 members within 6 months. We then actively engaged with these members following the underlying principles of agenda design by developing good social conditions between stakeholders, facilitating dialogue and encouraging respect for experiential knowledge. To do this, we provided communications material that introduced the work and perspectives of different groups, provided opportunities for online discussion forums through Twitter and online forums, and held face-to-face meetings and events for mixed groups with all stakeholders considered 'experts'.

Phase 2 involved consultation. We prepared a simple anonymous online survey for stakeholders that asked individuals to propose research questions they felt need to be answered to advance practice in the field. We split responses into three sections: questions relating to the effects of SCCE, the content and components of assets or activities and the delivery and provision of assets and activities. This received 135 responses. We supplemented this survey with feedback from eight special interest groups that had organically developed among network members, and ideas put forward during two face-to-face events, each involving 90-100 participants.

Phase 3 involved integration and used a modified Delphi approach. ${ }^{29}$ The responses from phase 2 were grouped thematically into an initial draft agenda that was then put to the 93 founding members of the network. They responded to the draft with further suggestions and clarifications, and an iterative process followed until members were happy with the draft.

Phase 4 then involved returning the agenda to all network members and asking them to vote on which questions were of highest priority to each person's area of work. For this survey, we asked members to state which stakeholder group they represented. On completion, participants were given the option of re-answering the survey assuming a different role (e.g. first as a researcher and then as an individual with lived experience), and 15 participants opted to do so. In total, 284 responses were received in this phase, as shown in Table 2.

This work received ethical approval from the University College London Research Ethics Committee (approval number: 14895/004), and all involved in the agenda process gave written or verbal informed consent.

\section{Results}

\section{The research agenda}

We identified four core themes within the agenda: the mode of engagement, the process of engagement, the impact of engagement and the infrastructure required to facilitate engagement (see Fig. 1). The full list of research questions in the agenda is shown in the

\begin{tabular}{|c|c|c|}
\hline Stakeholder group & $N$ & $\%$ \\
\hline Psychiatrists, other health professionals or trainees & 45 & 15.7 \\
\hline $\begin{array}{l}\text { Researcher (including PhD students and early-career } \\
\text { researchers) }\end{array}$ & 92 & 32.2 \\
\hline $\begin{array}{l}\text { Individuals with lived experience, lived experience groups and } \\
\text { members of the public }\end{array}$ & 55 & 19.9 \\
\hline $\begin{array}{l}\text { Representatives from community and third-sector } \\
\text { organisations }\end{array}$ & 59 & 20.6 \\
\hline Individuals working within policy, commissioning or strategy & 33 & 11.5 \\
\hline
\end{tabular}

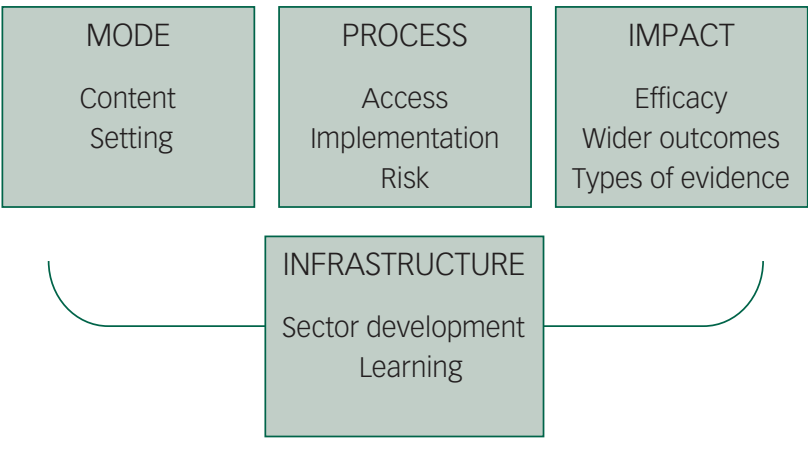

Fig. 1 Summary of themes in the research agenda.

supplementary material (Appendix 1) available at https://oi.org/ 10.1192/bjo.2020.133 and summarised below.

In relation to mode of engagement, two themes were identified. First, a number of research questions emerged relating to the content of community activities or engagement with community assets. These include questions on whether all types of SCCE are equally beneficial for mental health, if some modes of SCCE (e.g. live versus digital, or alone versus in groups) are more effective than others, whether certain types of facilitation or leadership of engagement are more effective than others, what amount of engagement is most beneficial for mental health, if quality of asset or activity content affects outcomes, what makes community activities and assets attractive for participants to engage and how activities and assets can be co-designed to meet the needs of individuals. There were also two main questions relating to the setting of SCCE: whether there is a difference in delivery and effectiveness depending on where engagement takes place, and exploring how 'place' shapes outcomes (for detailed research questions within these themes see Supplementary Appendix 1, sections 1-2).

In relation to the process of engagement, three themes were identified. A number of research questions emerged relating to access: how patterns of SCCE vary depending on individual characteristics, what the barriers or enablers to SCCE are among different groups and how we can create effective referral pathways to SCCE. There were also questions relating to implementation: whether activities and assets are being implemented appropriately, whether some people benefit more than others and what economic, political, social and cultural conditions are required for activities or assets to be a success. Finally, there were questions relating to risk: how we can minimise potential unintended consequences or adverse events arising from community activities, and how we can ensure safety and safeguarding of participants (for detailed research questions within these themes see Supplementary Appendix 1, sections 3-5).

In relation to impact of engagement, three themes were identified. Questions emerged relating to efficacy: the need for research exploring the effects of SCCE on prevention of mental illness and well-being among healthy individuals; research exploring the effects of SCCE on individuals with mild-to-moderate mental illness; research exploring the effects of SCCE for individuals with serious mental illness; and research exploring the effects of SCCE on the mental health of those delivering activities. There were also research questions on: wider outcomes, including under-researched outcomes related to mental health; secondary outcomes such as physical health, social determinants or behavioural risk factors; mechanisms by which SCCE affects mental health, comparing outcomes with those from non-SCCE interventions; and economic benefits. Finally, there were questions on the types of evidence being used: the use of under-utilised study designs such as ethnographies; analyses of population- 
level data and large-scale clinical trials; applying cross-disciplinary methods such as ecological monitoring, machine learning or geographical mapping; developing new theory to frame research on SCCE; using routinely collected data on health, education or behaviours; and comparing findings across different studies (e.g. through meta-analyses or developing standardised metrics or comparable measures for use across studies) (for detailed research questions within these themes see Supplementary Appendix 1, sections 6-8).

Finally, in relation to infrastructure required, two themes were identified. There were questions on sector development, including how we can train and support facilitators and practitioners, how we can support community organisations to work in this space, how we can expand the delivery of community activities and assets, how community activities can be made 'sustainable' for the future and how we could influence future policy developments to support this area of work. There were also questions on learning, including on how we can communicate better with the public, how we can share research better and how we can plan for new development opportunities with different sectors (for detailed research questions within these themes see Supplementary Appendix 1, sections 9-10).

\section{Prioritisation of research}

We created 'heat maps' showing which specific questions within the themes outlined above received the highest numbers of votes from different stakeholder groups (see Supplementary Appendix 2). There were many points of agreement across all stakeholder groups, such as the importance of co-production within design, the need to undertake more efficacy research and the importance of how to make SCCE 'sustainable' for the future. Further, all groups gave relatively low priority to research into new types of evidence (with the exception of theory development among psychiatrists and other health professionals, researchers and policy makers), suggesting an agreement on the importance of prioritising research with immediate relevance to outcomes if we are to improve practice.

However, there were also noticeable differences (see Supplementary Appendix 2). Among psychiatrists and other health professionals, and community and third-sector organisations, there was particular interest in infrastructure, whereas among individuals working within policy, commissioning and strategy, there was particular interest in the effects of place on outcomes, the economic, political, social and cultural conditions required for activities or assets to be a success, and the economic benefits. Among individuals with lived experience and members of the public, there was also interest in how much engagement was most beneficial to mental health. In addition, this group along with researchers and representatives from community organisations highlighted the importance of research on how to train and support facilitators and practitioners.

\section{Discussion}

This agenda confirms a number of the core themes that have emerged from previous high-level research agendas, including the UK Research and Innovation agenda, such as a focus on social and contextual factors, explorations of protective and resilience factors, improvements in public awareness and understanding, elucidation of mechanisms of action, considerations of place and participation, and barriers and enablers of engagement among different groups. ${ }^{21}$ However, it moves beyond these previous high-level agendas by seeing research on SCCE not just as a subset of a larger research agenda, but as an overarching topic that has its own specific research questions within it. Some of our priorities align with research recommendations from the National Institute for Health and Care Excellence on community engagement for improving health and well-being, including a focus on effectiveness (and cost-effectiveness, as prioritised by our policy stakeholders), a consideration of mechanisms and theory (as prioritised by our researchers and by psychiatrists and other health professionals) and a focus on collaborations and partnerships that can enable such work (as outlined in our sector development questions). ${ }^{30}$ The findings regarding barriers and enablers are also in line with recommendations in the updated Marmot Review, which recognises the importance of SCCE and community assets in addressing health inequalities, ${ }^{31}$ and the importance of SCCE in childhood is highlighted across a number of themes, echoing calls both from the Marmot Review and the World Health Organization's Social Determinants of Mental Health review. ${ }^{31,32}$ Further, our identification of questions relating to design, delivery and infrastructure is relevant given research suggesting that the majority of research in community-health sciences is skewed toward outcomes. ${ }^{33}$ These broader questions are therefore particularly highlighted as research gaps. Overall, our prioritisation exercise suggests there is a general consensus on the importance of key areas of research around efficacy, co-design and sustainability. However, it also highlights specific nuances among different stakeholders, suggesting the importance of considering the needs and requirements of different groups in developing research proposals or funding strategies. The agenda proposed is deliberately rich and diverse as different funders, research groups and stakeholders all have different areas of priority themselves. Therefore, different research questions in this agenda will speak to different groups. Nevertheless, the presentation of these diverse questions alongside one another in a unified research agenda should help future research to be more coordinated, and help to highlight the importance of considering different research questions (from the underlying research infrastructure to the tangible research impact) in parallel if we are truly to advance work in this field and the importance of further research into translation and innovation pathways.

Key strengths of the development of this research agenda include its open participatory, co-designed approach involving a large number of different stakeholders; the use of a range of modalities to elicit research questions from individuals; the use of a consensus approach to arrive at the final agenda; and the prioritisation of different research questions by different stakeholder groups. However, there are some limitations that affect the generalisability of the agenda presented. First, this work focused specifically on the UK context, so all stakeholders were from Great Britain or Northern Ireland. This enabled specificity when considering which research questions should be prioritised to inform practice, but it may mean that there are different or additional research questions that could or should be prioritised in other countries. Second, in prioritising research questions, stakeholders were asked to consider what would make the greatest difference to practice. Therefore, this prioritisation does not necessarily imply that those questions receiving lower votes are less important overall. Different funders may wish to use the agenda questions in new rounds of prioritisation to consider other priorities such as the advancement of research understanding, which may lead to higher prioritisation of those questions relating to topics such as types of evidence. Relatedly, this agenda specifically focuses on research, or how research can inform design and delivery. There may be other aspects to design and delivery and the broader environment that are priorities within practice or policy, but are not best explored through research, and therefore will not be captured within this agenda. In addition, the agenda focuses on where the key gaps are for future research, so areas that are already thought to be well covered are not shown. Finally, this agenda will inevitably 
evolve as new research is undertaken and as new challenges emerge. Therefore, it is intended to be a starting point for researchers and research funders rather than a constraint.

This agenda is timely as it considers the role of non-medical activities and assets within communities in supporting the prevention and management of mental illness. In the wake of the current COVID-19 pandemic, it is anticipated that there will be extreme pressure on mental health services in the coming months and years, leading to concerns that existing services will be unable to cope with demand. It is therefore of paramount importance that opportunities for providing mental health support from other sectors (including the cultural and community sectors) are properly explored to maximise the resources available. But it is also critical that the provision of such support remains evidence-based: that individuals are referred to appropriate, effective interventions and that the process of these referrals is transparent and suitable; and that there is sufficient infrastructure in place to support the organisations delivering social, cultural and community assets and activities. This research agenda identifies which research questions are going to be of highest priority in that effort. The agenda has now been taken forward within the research network to phases 5 and 6 of the agenda-developing process outlined by Abma and Broerse, ${ }^{34}$ which have involved developing a programme based on the research agenda and implementing the agenda through providing research funding targeted at the highest priority research questions presented. However, these phases should not be limited to the work of a single network, and so the agenda is presented here for researchers to continue implementing schemes that act on the findings. It is hoped that this will support the development of high-impact research within this valuable field of enquiry.

Daisy Fancourt $(\mathbb{D}$, Department of Behavioural Science and Health, University College London, UK: Kamaldeep Bhui (DD), Centre for Psychiatry, Barts \& The London School of Medicine and Dentistry, Queen Mary University of London, UK; Helen Chatterjee, Research Department of Genetics, Evolution and Environment, Division of Biosciences, University College London, UK; Paul Crawford, Institute of Mental Health, University o Nottingham, UK; Geoffrey Crossick, School of Advanced Study, University of London, UK; Tia DeNora, Department of Sociology, University of Exeter, UK; Jane South, Schoo of Health \& Community Studies, Leeds Beckett University, UK

Correspondence: Dr Daisy Fancourt. Email: d.fancourt@ucl.ac.uk

First received 15 Apr 2020, final revision 12 Oct 2020, accepted 15 Oct 2020

\section{Supplementary material}

Supplementary material is available online at https://doi.org/10.1192/bjo.2020.133.

\section{Data availability}

The data that support the findings of this study are available from the corresponding author, D.F., upon reasonable request.

\section{Author contributions}

All authors were involved in the design of the process and contributed to the research agenda development. D.F. drafted the manuscript and all authors critically reviewed and approved for submission.

\section{Funding}

The work was carried out as part of the MARCH Mental Health Network, funded by the CrossDisciplinary Mental Health Network Plus initiative supported by UK Research and Innovation (grant ES/S002588/1)

\section{Declaration of interest}

\section{References}

1 Morgan A, Ziglio E. Revitalising the evidence base for public health: an assets model. Promot Educ 2007; 14(suppl 2): 17-22.

2 Public Health England. A Guide to Community-Centred Approaches for Health and Wellbeing. Public Health England, 2015 (https://www.gov.uk/government/ publications/health-and-wellbeing-a-guide-to-community-centred-approaches).

3 Hopkins T, Rippon S. Head, Hands and Heart: Asset-Based Approaches in Health Care. Health Foundations, 2015.

4 Dodd F, Graves A, Taws K. Our Creative Talent - The Voluntary and Amateur Arts in England. Department for Culture, Media and Sport \& Arts Council England, 2013 (http://www.culturehive.co.uk/resources/our-creative-talentthe-voluntary-and-amateur-arts-in-england/).

5 Bagnall A-M. Places, Spaces, People and Wellbeing: A Systematic Review of Interventions to Boost Social Relations through Improvements in Community Infrastructure (Places and Spaces). What Works Centre for Wellbeing, 2018 (https://whatworkswellbeing.org/product/places-spaces-people-and-wellbeing/).

6 Daykin N, Mansfield L, Meads C, Julier G, Tomlinson A, Payne A, et al. What works for wellbeing? A systematic review of wellbeing outcomes for music and singing in adults. Perspect Public Health 2018; 138(1): 39-46.

7 Fancourt D, Finn S. What is the evidence on the role of the arts in improving health and well-being? A scoping review. WHO Regional Office for Europe, 2019 (Health Evidence Network (HEN) synthesis report 67).

8 Jenkinson CE, Dickens AP, Jones $\mathrm{K}$, Thompson-Coon J, Taylor RS, Rogers $\mathrm{M}$ et al. Is volunteering a public health intervention? A systematic review and meta-analysis of the health and survival of volunteers. BMC Public Health 2013; 13: 773.

9 Mansfield L, Kay T, Meads C, Grigsby-Duffy L, Lane J, John A, et al. Sport and dance interventions for healthy young people (15-24 years) to promote subjective well-being: a systematic review. BMJ Open 2018; 8(7): e020959.

10 Pennington A, Jones R, Bagnal A-M, South J, Cocoran R. The impact of historic places and assets on community wellbeing-A scoping review. What Works Centre for Wellbeing, 2019 (https://livrepository.liverpool.ac.uk/3034624/1/ Heritage-scoping-review-March-2019.pdf).

11 Tomlinson A. A Systematic Review of the Subjective Wellbeing Outcomes of Engaging with (Taking Part in, Performing, Viewing) Visual Arts for 'WorkingAge' Adults (15-64 Years) with Diagnosed Mental Health Conditions. What Works Centre for Wellbeing, 2018 (https://arro.anglia.ac.uk/id/eprint/702832/).

12 Jenkinson CE, Dickens AP, Jones K, Thompson-Coon J, Taylor RS, Rogers M, et al. Is volunteering a public health intervention? A systematic review and meta-analysis of the health and survival of volunteers. BMC Public Health 2013; 13: 773

13 Moss S. Natural Childhood. National Trust, 2012 (https://nt.global.ssl.fastly.net/ documents/read-our-natural-childhood-report.pdf).

14 Cultural Utilities Enterprises. The Alchemy Project Evaluation Report. Optimity Advisors, 2016 (https://www.gsttcharity.org.uk/sites/default/files/Final\%20 Report\%20-\%20The\%20Alchemy\%20Project\%20Evaluation\%2021\%2002\%20 2016.pdf).

15 Dowrick C, Billington J, Robinson J, Hamer A, Williams C. Get into reading as an intervention for common mental health problems: exploring catalysts for change. Med Humanit 2012; 38(1): 15-20.

16 Fancourt D, Aughterson $\mathrm{H}$, Finn $\mathrm{S}$, Walker $\mathrm{E}$, Steptoe A. How leisure activities affect health: a review and multi-level theoretical framework of mechanisms of action using the lens of complex adaptive systems science. Lancet Psychiatry 2020 (in press).

17 Alderwick HA, Gottlieb LM, Fichtenberg CM, Adler NE. Social prescribing in the US and England: emerging interventions to address patients' social needs. Am J Prev Med 2018; 54(5): 715-8.

18 Drinkwater C, Wildman J, Moffatt S. Social prescribing. BMJ 2019; 364: I1285.

19 Jensen A, Stickley T, Torrissen W, Stigmar K. Arts on prescription in Scandinavia: a review of current practice and future possibilities. Perspect Public Health 2017; 137(5): 268-74.

20 NHS England. The NHS Long Term Plan. NHS England, 2019 (https://www. longtermplan.nhs.uk/publication/nhs-long-term-plan/).

21 UK Research and Innovation. Widening Cross-Disciplinary Research for Mental Health: A Cross-Disciplinary Mental Health Research Agenda. UK Research and Innovation, 2017.

22 Department of Health London. A Framework for Mental Health Research. Department of Health London, 2017 (https://www.gov.uk/government/ publications/a-framework-for-mental-health-research).

23 Mental Health Taskforce to the NHS in England. The Five Year Forwards View for Mental Health. NHS England, 2016 (https://www.england.nhs.uk/wp-content/ uploads/2016/02/Mental-Health-Taskforce-FYFV-final.pdf). 
24 Medical Research Council. Strategy for Lifelong Mental Health Research. Medical Research Council, 2017 (https://mrc.ukri.org/documents/pdf/strategy-forlifelong-mental-health-research/)

25 Wellcome Trust. Mental Health Programme Strategy. Wellcome Trust, 2019 (https://wellcome.org/what-we-do/our-work/mental-health-transforming-research and-treatments/strategy).

26 Rose D, Carr S, Beresford P. 'Widening cross-disciplinary research for menta health': what is missing from the Research Councils UK mental health agenda? Disabil Soc 2018; 33(3): 476-81.

27 Broerse JE, Zweekhorst MB, van Rensen AJ, de Haan MJ. Involving burn survivors in agenda setting on burn research: an added value? Burns 2010; 36(2): 217-31.

28 Caron-Flinterman JF, Broerse JE, Teerling J, Van Alst ML, Klaasen S, Swart LE, et al. Stakeholder participation in health research agenda setting: the case of asthma and COPD research in the Netherlands. Sci Public Policy 2006; 33(4): 291-304.

29 Hsu C-C, Sandford BA. The Delphi technique: making sense of consensus. Practical assessment. Res Eval 2007; 12: 10.
30 National Institute of Health and Care Excellence (NICE). Recommendations for Research | Community Engagement: Improving Health and Wellbeing and Reducing Health Inequalities. NICE Guideline [NG44]. NICE, 2016 (https:// www.nice.org.uk/guidance/ng44/chapter/Recommendations-for-research).

31 Marmot M, Allen J, Boyce T, Goldblatt P, Morrison J. Health Equity in England: The Marmot Review 10 Years On. Institute of Health Equity, 2020 (https:/l www.health.org.uk/publications/reports/the-marmot-review-10-years-on).

32. World Health Organization (WHO), Calouste Gulbenkian Foundation. Social Determinants of Mental Health. WHO, 2014 (http://www.who.int/mental health/publications/gulbenkian_paper_social_determinants_of_mental_health/en/).

33 Greenhalgh $T$, Fahy $N$. Research impact in the community-based health sciences: an analysis of 162 case studies from the 2014 UK Research Excellence Framework. BMC Med 2015; 13(1): 232.

34 Abma TA, Broerse JEW. Patient participation as dialogue: setting research agendas. Health Expect 2010; 13(2): 160-73. 